\title{
Neutron skin in CsI and low-energy effective weak mixing angle from COHERENT data
}

\author{
Xu-Run Huang and Lie-Wen Chen $\odot^{*}$ \\ School of Physics and Astronomy and Shanghai Key Laboratory for Particle Physics and Cosmology, \\ Shanghai Jiao Tong University, Shanghai 200240, China
}

(Received 26 February 2019; published 7 October 2019)

\begin{abstract}
Both the neutron skin thickness $\Delta R_{n p}$ of atomic nuclei and the low-energy neutrino-nucleon $(\nu N)$ interactions are of fundamental importance in nuclear and particle physics, astrophysics as well as new physics beyond the standard model (SM) but largely uncertain currently, and the coherent elastic neutrino-nucleus scattering $(\mathrm{CE} \nu \mathrm{NS})$ provides a clean way to extract their information. New physics beyond the SM may cause effectively a shift of the SM weak mixing angle $\theta_{W}$ in low-energy $\nu N$ interactions, leading to an effective weak mixing angle $\theta_{W}^{*}$. By analyzing the CE $\nu$ NS data of the COHERENT experiment, we find that while a oneparameter fit to the COHERENT data by varying $\Delta R_{n p}$ produces $\Delta R_{n p}^{\mathrm{CsI}} \simeq 0.68_{-1.13}^{+0.91} \mathrm{fm}$ for CsI with an unrealistically large central value by fixing $\sin ^{2} \theta_{W}^{*}$ at the low-energy $\mathrm{SM}$ value of $\sin ^{2} \theta_{W}^{\mathrm{SM}}=0.23857$, a twodimensional fit by varying $\Delta R_{n p}$ and $\sin ^{2} \theta_{W}^{*}$ leads to a strong positive correlation between $\Delta R_{n p}$ and $\sin ^{2} \theta_{W}^{*}$ with significantly smaller central values of $\Delta R_{n p}^{\mathrm{CsI}} \simeq 0.24_{-2.03}^{+2.30} \mathrm{fm}$ and $\sin ^{2} \theta_{W}^{*}=0.21_{-0.10}^{+0.13}$. Although the uncertainty is too large to claim a determination of $\Delta R_{n p}^{\mathrm{CsI}}$ and $\sin ^{2} \theta_{W}^{*}$, the present study suggests that the multidimensional fit is important in future analyses of high-precision CE $\nu$ NS data. The implication of the possible deviation of $\sin ^{2} \theta_{W}^{*}$ from $\sin ^{2} \theta_{W}^{\mathrm{SM}}$ on new physics beyond the SM is also discussed.
\end{abstract}

DOI: 10.1103/PhysRevD.100.071301

\section{INTRODUCTION}

The neutron skin thickness of atomic nuclei, defined as $\Delta R_{n p}=R_{n}-R_{p}$ where $R_{n(p)}=\left\langle r_{n(p)}^{2}\right\rangle^{1 / 2}$ is the neutron (proton) rms radius of the nucleus, provides a good probe of the equation of state (EOS) for isospin asymmetric nuclear matter [1-10], which is critically important due to its multifaceted roles in nuclear physics and astrophysics [11-14] as well as some issues of new physics beyond the standard model (SM) [15-19]. While the $R_{p}$ can be measured precisely from electromagnetic processes (see, e.g., Refs. [20-22]), the $R_{n}$ is largely uncertain since it is usually determined from strong processes, which is generally model dependent due to the complicated nonperturbative effects. This provides a strong motivation for the lead radius experiment (PREX) being performed at the Jefferson Laboratory to determine the $R_{n}$ of ${ }^{208} \mathrm{~Pb}$ to about $1 \%$ accuracy by measuring the parity-violating electroweak asymmetry in the elastic scattering of polarized electrons from ${ }^{208} \mathrm{~Pb}$ [23]. The PREX Collaboration reported the first result of the parity violating weak neutral interaction measurement of the $\Delta R_{n p}$ for ${ }^{208} \mathrm{~Pb}$,

\footnotetext{
*Corresponding author: lwchen@sjtu.edu.cn
}

Published by the American Physical Society under the terms of the Creative Commons Attribution 4.0 International license. Further distribution of this work must maintain attribution to the author(s) and the published article's title, journal citation, and DOI. Funded by SCOAP. i.e., $\Delta R_{n p}^{208}=0.33_{-0.18}^{+0.16} \mathrm{fm}$ [24] (see, also, Ref. [25]). The central value of $0.33 \mathrm{fm}$ means a surprisingly large neutron skin thickness in ${ }^{208} \mathrm{~Pb}$ although there is no compelling reason to rule out a such large value [26].

Recently, the COHERENT Collaboration [27] reported the first observation of the coherent elastic neutrino-nucleus scattering $(\mathrm{CE} \nu \mathrm{NS})$ [28,29]. In Ref. [30], a value of the averaged $\Delta R_{n p}$ of ${ }_{55}^{133} \mathrm{Cs}$ and ${ }_{53}^{127} \mathrm{I}$, i.e., $\Delta R_{n p}^{\mathrm{CsI}} \simeq 0.7_{-1.1}^{+0.9} \mathrm{fm}$, is extracted from analyzing the COHERENT data. The extracted central value of $\Delta R_{n p}^{\mathrm{CsI}} \simeq 0.7 \mathrm{fm}$ is unrealistically large. To the best of our knowledge, $\Delta R_{n p}^{\mathrm{CsI}} \simeq 0.7 \mathrm{fm}$ is actually much larger than all the predictions of current nuclear models. Moreover, since ${ }^{208} \mathrm{~Pb}$ is much more neutron-rich than ${ }_{55}^{133} \mathrm{Cs}$ and ${ }_{53}^{127} \mathrm{I}$, the $\Delta R_{n p}^{\mathrm{CsI}}$ is expected to be smaller than the $\Delta R_{n p}^{208}$ according to the neutron skin systematics [31,32], and thus $\Delta R_{n p}^{\mathrm{CsI}} \simeq 0.7 \mathrm{fm}$ is inconsistent with the PREX result. Although the uncertainty is too large to claim a determination of $\Delta R_{n p}^{\mathrm{CSI}}$, the best-fit value $\Delta R_{n p}^{\mathrm{CSI}} \simeq$ $0.7 \mathrm{fm}$ indicates the possibility of a unrealistically large neutron skin thickness. The possible inconsistency could be a hint of new physics in neutrino physics and this provides the main motivation of the present work.

We note that in Ref. [30], the $\Delta R_{n p}^{\mathrm{CsI}}$ is extracted from a one-parameter fit to the COHERENT data by varying $\Delta R_{n p}^{\text {CsI }}$ with the low-energy weak mixing angle $\theta_{W}$ fixed at the SM value $\sin ^{2} \theta_{W}^{S M}=0.23865$ obtained in the modified minimal subtraction $(\overline{\mathrm{MS}})$ renormalization scheme at near 
zero momentum transfer $Q=0$ [33] (the newest value is $\sin ^{2} \theta_{W}^{\mathrm{SM}}=0.23857(5)$ [34]). Experimentally, the precise determination of $\sin ^{2} \theta_{W}$ at low $Q^{2}$ is an ongoing issue [35], and the atomic parity violation (APV) experiments offer the most precise results to date. For example, by measuring the $6 s_{1 / 2}-7 s_{1 / 2}$ electric dipole transition in ${ }^{133} \mathrm{Cs}$ atom, a value of $\sin ^{2} \theta_{W}=0.2356(20)$ at $\langle Q\rangle \simeq$ $2.4 \mathrm{MeV}$ is obtained [36-38], which is smaller than $\sin ^{2} \theta_{W}^{\mathrm{SM}}$ by about $1.5 \sigma$. In the midenergy regime, the Qweak Collaboration reported the recent measurement on proton's weak charge and obtained $\sin ^{2} \theta_{W}=0.2383(11)$ at $Q=0.158 \mathrm{GeV}$ [39], agreeing well with the SM prediction. On the other hand, the low-energy neutrino-nucleon $(\nu N)$ interactions could involve new physics beyond the SM [35,40-47], which may cause effectively a shift of the SM weak mixing angle $\theta_{W}$ in the $\nu N$ interactions, leading to a low-energy effective weak mixing angle $\theta_{W}^{*}$. Any experimental constraints on $\theta_{W}^{*}$ would provide useful information on new physics beyond the SM.

In this work, we extract the values of $\Delta R_{n p}^{\mathrm{CsI}}$ and $\sin ^{2} \theta_{W}^{*}$ using a two-dimensional (2D) fit to the COHERENT data by varying $\Delta R_{n p}^{\mathrm{CSI}}$ and $\sin ^{2} \theta_{W}^{*}$. Compared to the results using one-parameter fit with $\sin ^{2} \theta_{W}^{*}$ fixed at $\sin ^{2} \theta_{W}^{\mathrm{SM}}$, we find a strong positive correlation between $\Delta R_{n p}$ and $\sin ^{2} \theta_{W}^{*}$ with significantly smaller central values of $\Delta R_{n p}^{\text {CsI }} \simeq$ $0.24_{-2.03}^{+2.30} \mathrm{fm}$ and $\sin ^{2} \theta_{W}^{*}=0.21_{-0.10}^{+0.13}$ at $Q \simeq 0.05 \mathrm{GeV}$ (corresponding to the energy scale of COHERENT experiment), indicating that the $\sin ^{2} \theta_{W}^{*}$ may play an important role in extracting neutron skin information from analyzing the $\mathrm{CE} \nu \mathrm{NS}$ data.

\section{CE $\nu$ NS IN THE COHERENT EXPERIMENT}

The differential cross section for coherent elastic neutrino-nucleus scattering has a straightforward SM prediction in the case with different proton and neutron distributions (form factors) in the nucleus. By neglecting the radiative corrections and axial contributions, the cross section can be expressed as $[41,42,48-50]$ :

$$
\begin{aligned}
\frac{\mathrm{d} \sigma}{\mathrm{d} T}\left(E_{\nu}, T\right) & =\frac{G_{F}^{2} M}{2 \pi} G_{V}^{2}\left[1-\frac{M T}{E_{\nu}^{2}}+\left(1-\frac{T}{E_{\nu}}\right)^{2}\right], \\
G_{V} & =Z g_{V}^{p} F_{p}\left(q^{2}\right)+N g_{V}^{n} F_{n}\left(q^{2}\right),
\end{aligned}
$$

where $G_{F}$ is the Fermi coupling constant, $M$ is the nucleus mass, $E_{\nu}$ and $T$ are neutrino energy and nuclear recoil kinetic energy, respectively. For a given $E_{\nu}$, the corresponding $T$ varies from 0 to $T^{\max }=2 E_{\nu}^{2} /\left(M+2 E_{\nu}\right)$. The proton and neutron neutral current vector couplings are defined, respectively, as $g_{V}^{p}=\frac{1}{2}-2 \sin ^{2} \theta_{W}$ and $g_{V}^{n}=-\frac{1}{2}$. The form factor $F_{n(p)}\left(q^{2}\right)$ encapsulate the neutron (proton) number density distribution in nuclei, where the momentum transfer $q$ is given by $q^{2}=2 E_{\nu}^{2} T M /\left(E_{\nu}^{2}-E_{\nu} T\right) \simeq 2 M T$ under the condition of $E_{\nu} \gg T$.

In the case of the COHERENT experiment, the measurement is performed using a CsI detector which is dominantly composed of ${ }_{55}^{133} \mathrm{Cs}$ and ${ }_{53}^{127} \mathrm{I}$. The mass of a nucleus with $N(Z)$ neutrons (protons) is determined by its corresponding total binding energy $\left(E_{B}\right)$ from $M=$ $N \times m_{n}+Z \times m_{p}-E_{B}$ where $m_{n(p)}$ is the rest mass of neutrons (protons). The binding energies per nucleon are $8.40998 \mathrm{MeV}$ and $8.44549 \mathrm{MeV}$ [51] for isotopes ${ }^{133} \mathrm{Cs}$ and ${ }^{127} \mathrm{I}$, respectively. As for their density distributions, in order to test the model dependence, two analytic nuclear form factors are adopted, namely, the symmetrized Fermi (SF) form factor and the Helm form factor, which are two very successful and well-tested forms of nuclear form factors for medium to heavy nuclei [30,52-55]. Both form factors are characterized by two parameters related to the nuclear radius and the surface thickness (diffuseness), respectively.

The SF form factor has the form (See, e.g., Ref. [55])

$$
\begin{aligned}
F_{\mathrm{SF}}\left(q^{2}\right)= & \frac{3}{q c\left[(q c)^{2}+(\pi q a)^{2}\right]}\left[\frac{\pi q a}{\sinh (\pi q a)}\right] \\
& \times\left[\frac{\pi q a}{\tanh (\pi q a)} \sin (q c)-q c \cos (q c)\right],
\end{aligned}
$$

and the corresponding rms radius is expressed as

$$
R_{\mathrm{SF}}^{2} \equiv\left\langle r^{2}\right\rangle=\frac{3}{5} c^{2}+\frac{7}{5}(\pi a)^{2} .
$$

where $c$ is the half-density radius and $a$ quantifies the surface thickness $t=4 a \ln 3$. Experimentally, the proton distribution has been determined precisely, and we take the same parameters for proton distribution as in Ref. [30], which are obtained by fitting the proton structure data of ${ }^{133} \mathrm{Cs}$ and ${ }^{127} \mathrm{I}$ measured in muonic atom spectroscopy, namely, $t_{p}=2.30 \mathrm{fm}, \quad c_{p, \mathrm{Cs}}=5.6710 \pm 0.0001 \mathrm{fm}$ and $c_{p, \mathrm{I}}=$ $5.5931 \pm 0.0001 \mathrm{fm}$. The corresponding proton rms radii for ${ }^{133} \mathrm{Cs}$ and ${ }^{127} \mathrm{I}$ are $R_{p}^{\mathrm{Cs}}=4.804 \mathrm{fm}$ and $R_{p}^{\mathrm{I}}=4.749 \mathrm{fm}$, respectively.

The Helm form factor is expressed as [52]

$$
F_{\text {Helm }}\left(q^{2}\right)=3 \frac{j_{1}\left(q R_{0}\right)}{q R_{0}} \mathrm{e}^{-q^{2} s^{2} / 2},
$$

where $j_{1}(x)$ is the spherical Bessel function of order one, i.e., $j_{1}(x)=\sin (x) / x^{2}-\cos (x) / x$. The rms radius is simply given by

$$
R_{\text {Helm }}^{2} \equiv\left\langle r^{2}\right\rangle=\frac{3}{5} R_{0}^{2}+3 s^{2},
$$

where $R_{0}$ is the box radius and $s$ quantifies the surface thickness. Again, for the proton distributions in ${ }^{133} \mathrm{Cs}$ and ${ }^{127} \mathrm{I}$, we use $s_{p}=0.9 \mathrm{fm}$ following Ref. [30], which 
was determined for the proton form factor of similar nuclei [56], and the $R_{0, p}$ is determined by the corresponding $R_{p}$.

For the parameters of the neutron distributions in ${ }^{133} \mathrm{Cs}$ and ${ }^{127} \mathrm{I}$, they are essentially unknown. In these neutron-rich nuclei, in principle, the neutron distributions should be different from the proton distributions because of the charge difference, which means that the neutron distributions could have different radius parameters $\left(c_{n}\right.$ and $\left.R_{0, n}\right)$ and diffuseness (surface thickness) parameters $\left(t_{n}\right.$ and $\left.s_{n}\right)$ compared to the proton distributions. We will examine these effects in the following.

In the COHERENT experiment, the photoelectrons are counted to monitor the scattering events and extract the nuclear recoil energy, with approximately 1.17 photoelectrons expected per $\mathrm{keV}$ of nuclear recoil energy, denoted as $\zeta=1.17 \mathrm{keV}^{-1}$ [27]. The number of event counts in a nuclear recoil energy bin $\left[T^{i}, T^{i+1}\right]$ can be obtained as

$$
\begin{aligned}
N_{i}^{\mathrm{th}}= & N_{\mathrm{CsI}} \sum_{\nu_{l}} \sum_{\mathcal{N}=\mathrm{Cs}, \mathrm{I}} \int_{T^{i}}^{T^{i+1}} \mathrm{~d} T \mathcal{A}(\zeta T) \\
& \times \int_{E_{\nu}^{\min }}^{E_{\nu}^{\max }} \mathrm{d} E_{\nu} \frac{\mathrm{d} N_{\nu_{l}}}{\mathrm{~d} E_{\nu}} \frac{\mathrm{d} \sigma_{\nu-\mathcal{N}}}{\mathrm{d} T}
\end{aligned}
$$

where $N_{\text {CsI }}$ is the number of CsI in the detector and is given by $N_{A} m_{\text {det }} / M_{\text {CsI }}$ with $N_{A}$ being the Avogadro constant, $m_{\mathrm{det}}=14.57 \mathrm{~kg}$ the detector mass and $M_{\mathrm{CsI}}=$ $259.8 \mathrm{~g} / \mathrm{mol}$ the molar mass of CsI. The acceptance efficiency function $\mathcal{A}(x)$ is described by [57]

$$
\mathcal{A}(x)=\frac{a}{1+\mathrm{e}^{-k\left(x-x_{0}\right)}} \Theta(x-5)
$$

where the parameter values are taken as $a=0.6655_{-0.0384}^{+0.0212}$, $k=0.4942_{-0.0131}^{+0.0335}$ and $x_{0}=10.8507_{-0.3995}^{+0.1838}$, and the $\Theta(x)$ is a modified Heaviside step function defined as

$$
\Theta(x-5)= \begin{cases}0 & x<5 \\ 0.5 & 5 \leq x<6 \\ 1 & x \geq 6\end{cases}
$$

The value of $E_{\nu}^{\min }$ depends on $T$, and the $E_{\nu}^{\max }$ is related to the neutrino source. At the Spallation Neutron Source, the neutrino flux is generated from the stopped pion decays $\pi^{+} \rightarrow \mu^{+}+\nu_{\mu}$ as well as the subsequent muon decays $\mu^{+} \rightarrow e^{+}+\bar{\nu}_{\mu}+\nu_{e}$. The neutrino population has the following energy distributions $[30,43]$

$$
\begin{aligned}
\frac{\mathrm{d} N_{\nu_{\mu}}}{\mathrm{d} E_{\nu}} & =\eta \delta\left(E_{\nu}-\frac{m_{\pi}^{2}-m_{\mu}^{2}}{2 m_{\pi}}\right), \\
\frac{\mathrm{d} N_{\bar{\nu}_{\mu}}}{\mathrm{d} E_{\nu}} & =\eta \frac{64 E_{\nu}^{2}}{m_{\mu}^{3}}\left(\frac{3}{4}-\frac{E_{\nu}}{m_{\mu}}\right), \\
\frac{\mathrm{d} N_{\nu_{e}}}{\mathrm{~d} E_{\nu}} & =\eta \frac{192 E_{\nu}^{2}}{m_{\mu}^{3}}\left(\frac{1}{2}-\frac{E_{\nu}}{m_{\mu}}\right),
\end{aligned}
$$

with $E_{\nu}^{\max } \leq m_{\mu} / 2$. The normalization factor $\eta$ is defined as $\eta=r N_{\text {POT }} /\left(4 \pi L^{2}\right)$, where $r=0.08$ is the averaged production rate of the decay-at-rest (DAR) neutrinos for each flavor per proton on target, $N_{\text {POT }}=1.76 \times 10^{23}$ is the total number of protons delivered to the target and $L=19.3 \mathrm{~m}$ is the distance between the neutrino source and the CsI detector [27].

To evaluate the fitting quality on the COHERENT data in Fig. 3A of Ref. [27], following Ref. [30], we apply the following least-squares function with only the 12 energy bins from $i=4$ to $i=15$, i.e.,

$$
\begin{aligned}
\chi^{2}= & \sum_{i=4}^{15}\left(\frac{N_{i}^{\exp }-(1+\alpha) N_{i}^{\mathrm{th}}-(1+\beta) B_{i}}{\sigma_{i}}\right)^{2} \\
& +\left(\frac{\alpha}{\sigma_{\alpha}}\right)^{2}+\left(\frac{\beta}{\sigma_{\beta}}\right)^{2} .
\end{aligned}
$$

Here for each energy bin, the experimental number of events, denoted as $N_{i}^{\text {exp }}$, is generated from the C-AC differences, and $B_{i}$ is the estimated beam-on background with only prompt neutrons included [27]. The $\sigma_{i}=$ $\sqrt{N_{i}^{\mathrm{exp}}+2 B_{i}^{\mathrm{sS}}+B_{i}}$ is the statistical uncertainty where $B_{i}^{\text {ss }}$ is the estimated steady-state background determined with AC data [27]. The $\alpha$ and $\beta$ are the systematic parameters corresponding to the uncertainties on the signal rate and the beam-on background rate, respectively. The fractional uncertainties corresponding to $1-\sigma$ variation are $\sigma_{\alpha}=0.28$ and $\sigma_{\beta}=0.25$ [27]. All the experimental data are taken from the COHERENT release [57].

\section{RESULTS AND DISCUSSIONS}

In the present work for $\mathrm{CE} \nu \mathrm{NS}$ calculations, we replace the $\theta_{W}$ in Eq. (1) by $\theta_{W}^{*}$ to effectively consider the possible effects of new physics in $\nu N$ interactions. We first assume that the neutron and proton distributions have the same diffuseness parameters (i.e., $t_{n}=t_{p}$ and $s_{n}=s_{p}$ ) and the value of $\sin ^{2} \theta_{W}^{*}$ is fixed at the SM value of $\sin ^{2} \theta_{W}^{\mathrm{SM}}=0.23857$, and then perform a one-parameter fit to the COHERENT data by varying $R_{n}$ to extract the neutron rms radius $R_{n}^{\mathrm{CsI}}$ of CsI ${ }_{55}^{133} \mathrm{Cs}$ and ${ }_{53}^{127} \mathrm{I}$ are assumed to have equal $R_{n}$ ). Our calculations lead to $R_{n}=$ $5.46_{-1.13}^{+0.91} \mathrm{fm}$ with the Helm form factor and $R_{n}=$ $5.47_{-1.13}^{+0.91} \mathrm{fm}$ with the SF form factor. Our results thus 
nicely confirm the value of $R_{n}=5.5_{-1.1}^{+0.9} \mathrm{fm}$ extracted in Ref. [30] with the same assumptions.

In addition, we explore the effects of the neutron diffuseness parameters. To this end, we perform a oneparameter fit to the COHERENT data by varying $R_{n}^{\mathrm{CsI}}$ with various fixed values of the diffuseness parameter while the effective weak mixing angle is fixed at $\sin ^{2} \theta_{W}^{*}=\sin ^{2} \theta_{W}^{\mathrm{SM}}$. The results indicate that a variation of $\pm 0.02 \mathrm{fm}$ for $\Delta R_{n p}^{\mathrm{CsI}}$ arises when $s_{n}$ changes from 0.63 to $1.17 \mathrm{fm}$ (corresponding to a variation of $\pm 30 \%$ for $s_{n}=0.9 \mathrm{fm}$ ) in the Helm form factor. The same conclusion is obtained when the SF form factor is used. Therefore, compared to the obtained neutron skin thickness of $\Delta R_{n p}^{\mathrm{CsI}} \simeq 0.68_{-1.13}^{+0.91} \mathrm{fm}$, the effects of the neutron diffuseness parameters are indeed quite small, consistent with the statement in Ref. [30].

Now we turn to examining the effects of the low-energy effective weak mixing angle. The possible nonstandard running of $\sin ^{2} \theta_{W}^{*}$ in low-energy regime is expected to influence the extraction of the neutron distribution from the low-energy CE $\nu$ NS experiments. The simultaneous precise determination of the neutron distribution and the low-energy $\sin ^{2} \theta_{W}^{*}$ through $\mathrm{CE} \nu \mathrm{NS}$ experiments can (in)validate our knowledge of nuclear physics and neutrino physics. Hence, we perform a $2 \mathrm{D}$ fit to the COHERENT data by varying $R_{n}$ and $\sin ^{2} \theta_{W}^{*}$ using the Helm form factor with $s_{n}=s_{p}$. The resulting number of CE $\nu$ NS event counts as a function of the number of photoelectrons is shown in Fig. 1 while the corresponding $\chi^{2}$ contours are displayed in Fig. 2.

For comparison, we also include in Fig. 1 the corresponding results from the COHERENT data, the similar $2 \mathrm{D}$ fit by using the SF form factor with $t_{n}=t_{p}$, and the

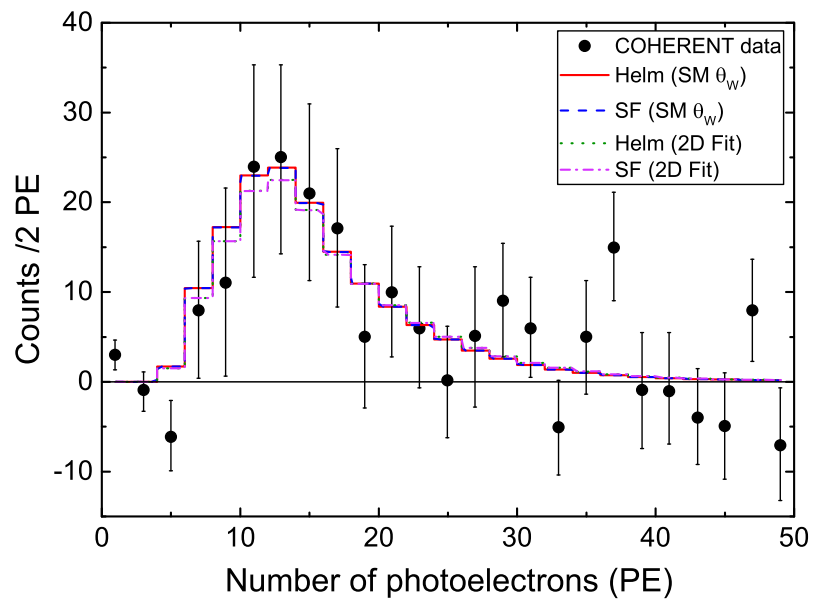

FIG. 1. The CE $\nu$ NS event counts as a function of the number of photoelectrons in the COHERENT experiment. The solid (dashed) line shows the results with best-fit neutron rms radius using the Helm (SF) form factor in the one-parameter fit when the $\sin ^{2} \theta_{W}^{*}$ is fixed at the SM prediction. The dotted (dash-dotted) line shows the results with best-fit parameters in the 2D fit using the Helm (SF) form factor. Data are taken from Ref. [27].

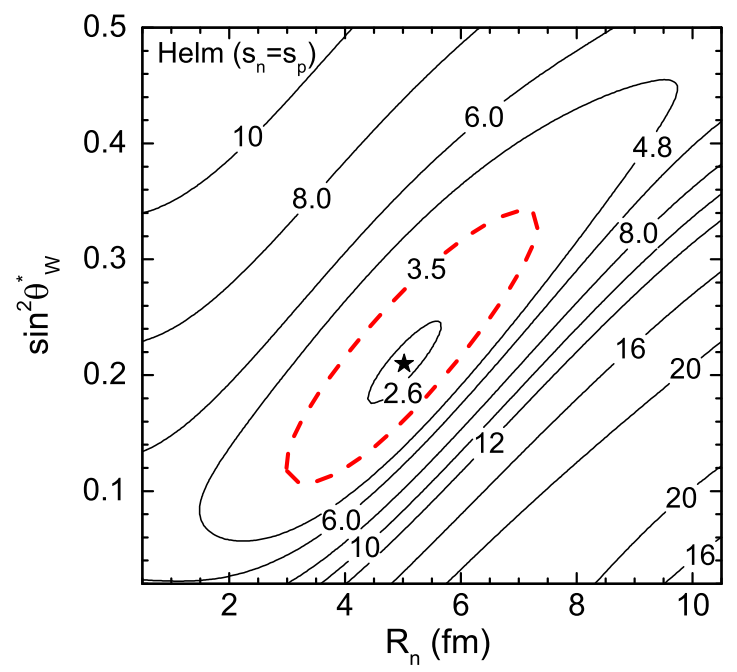

FIG. 2. The $\chi^{2}$ contours in the plane of $R_{n}$ vs $\sin ^{2} \theta_{W}^{*}$ obtained from a $2 \mathrm{D}$ fit to the COHERENT data using the Helm form factor with $s_{n}=s_{p}$. The star marks the center values of $R_{n}=5.02 \mathrm{fm}$ and $\sin ^{2} \theta_{W}^{*}=0.21$ at $\chi_{\min }^{2}=2.498$. The dashed curve corresponds to the contour at $\chi^{2}=\chi_{\min }^{2}+1$.

one-parameter fit by varying $R_{n}$ with fixed $\sin ^{2} \theta_{W}^{*}=$ $\sin ^{2} \theta_{W}^{\mathrm{SM}}$ using both the Helm and SF form factors. It is seen from Fig. 1 that for both one-parameter and 2D fits, the SF and Helm form factors produce almost identical results, indicating the independence of our results on the form of nuclear form factors. Furthermore, Fig. 1 indicates that compared to the one-parameter fit, the $2 \mathrm{D}$ fit predicts a fewer event counts in the region of $7-15$ for the photoelectron number, leading to a decrease by $\sim 3.2 \%$ for the number of total event counts.

From Fig. 2, one sees clearly that there exhibits a strong positive correlation between $R_{n}$ and $\sin ^{2} \theta_{W}^{*}$. Particularly interesting is that there exists favored center values for $R_{n}$ and $\sin ^{2} \theta_{W}^{*}$, i.e.,

$$
R_{n}^{\text {Helm }}=5.02_{-2.03}^{+2.30} \mathrm{fm}, \quad \sin ^{2} \theta_{W}^{*}=0.21_{-0.10}^{+0.13} .
$$

We note that very similar results are obtained when the SF form factor is used. With the averaged rms radii of protons and neutrons in ${ }^{133} \mathrm{Cs}$ and ${ }^{127} \mathrm{I}$, we then obtain the averaged neutron skin thickness of CsI as

$$
\Delta R_{n p}^{\mathrm{CsI}} \simeq 0.24_{-2.03}^{+2.30} \mathrm{fm}
$$

The favored central value $\Delta R_{n p}^{\mathrm{CsI}} \simeq 0.24 \mathrm{fm}$ is significantly smaller than $\Delta R_{n p}^{\mathrm{CsI}} \simeq 0.68 \mathrm{fm}$ extracted from the oneparameter fit to the COHERENT data with fixed $\sin ^{2} \theta_{W}^{*}=\sin ^{2} \theta_{W}^{\mathrm{SM}}$, indicating the importance of the $\sin ^{2} \theta_{W}^{*}$ in the extraction of $\Delta R_{n p}^{\mathrm{CsI}}$ from CE $\nu \mathrm{NS}$.

Furthermore, we examine the effects of neutron diffuseness parameters using the $2 \mathrm{D}$ fit to the COHERENT data by varying $R_{n}$ and $\sin ^{2} \theta_{W}^{*}$ with $s_{n}$ and $t_{n}$ fixed at various 
values. Our results indicate that the central value of $\Delta R_{n p}^{\mathrm{CsI}}$ varies by $\pm 0.03 \mathrm{fm}$ (the corresponding $R_{n}$ varies from $4.99 \mathrm{fm}$ to $5.05 \mathrm{fm}$ ) when the value of $s_{n}$ in the Helm form factor changes from $0.63 \mathrm{fm}$ to $1.17 \mathrm{fm}$ (corresponding to a variation of $\pm 30 \%$ for $s_{n}=0.9 \mathrm{fm}$ ). Similarly, we find the central value of $\Delta R_{n p}^{\text {CsI }}$ varies by $\pm 0.04 \mathrm{fm}$ (the corresponding $R_{n}$ varies from $4.99 \mathrm{fm}$ to $5.07 \mathrm{fm}$ ) when the value of $t_{n}$ in the SF form factor changes from $1.61 \mathrm{fm}$ to $2.99 \mathrm{fm}$ (corresponding to a variation of $\pm 30 \%$ for $t_{n}=2.3 \mathrm{fm}$ ). Meanwhile, we note the central value variation of $\sin ^{2} \theta_{W}^{*}$ is tiny, namely, from 0.209 to 0.211 when $s_{n}\left(t_{n}\right)$ changes from $0.63(1.61) \mathrm{fm}$ to $1.17(2.99) \mathrm{fm}$. The variation of $\pm(0.03-0.04) \mathrm{fm}$ is appreciable compared to the central value $\Delta R_{n p}^{\mathrm{CsI}} \simeq 0.24 \mathrm{fm}$, implying that one may extract useful information on the neutron diffuseness parameters in atomic nuclei from analyzing the future high-precise data of CELNS via a three-dimensional fit by varying $\sin ^{2} \theta_{W}^{*}$, $\Delta R_{n p}^{\mathrm{CsI}}$ and the diffuseness parameters $\left(s_{n}\right.$ and $\left.t_{n}\right)$. This can help to address the interesting question about whether the neutron skin structure is really from the bulk radius difference or the surface diffuseness difference between the neutron and proton distributions in atomic nuclei $[31,58,59]$. Therefore, our results suggest that a multidimensional fit is important to extract the value of $\sin ^{2} \theta_{W}^{*}$ and the neutron skin information including its size (i.e., $\Delta R_{n p}^{\mathrm{CsI}}$ ) and shape (e.g., $s_{n}$ and $t_{n}$ ) in future analyses of highprecision $\mathrm{CE} \nu \mathrm{NS}$ data. Nevertheless, the extracted central value of $\Delta R_{n p}^{\mathrm{CsI}} \simeq 0.24 \mathrm{fm}$ with an uncertainty of $\pm(0.03-0.04)$ fm obtained in the present work is consistent with some carefully calibrated nuclear models (see, e.g., Refs. [26,30]).

On the other hand, a possible substantial deviation of $\sin ^{2} \theta_{W}^{*}$ from $\sin ^{2} \theta_{W}^{\mathrm{SM}}$, i.e., $\Delta \sin ^{2} \theta_{W}^{*}=-0.02857$, is obtained with the best-fit value of $\sin ^{2} \theta_{W}^{*}=0.21$. This deviation could be a hint of new physics beyond SM in neutrino physics. For example, one new physics scenario is to introduce the nonstandard interactions (NSIs) in the SM interactions, which has been widely discussed [40-44]. To make a rough estimate on the parameters in NSIs, we introduce an ad hoc nonstandard charge $G_{V}^{\mathrm{NSI}}$ to replace the $G_{V}$ in Eq. (2), i.e.,

$$
\begin{aligned}
G_{V}^{\mathrm{NSI}}= & Z g_{V}^{p} F_{p}\left(q^{2}\right)+N g_{V}^{n} F_{n}\left(q^{2}\right) \\
& +3 \delta_{\mathrm{NSI}}\left[Z F_{p}\left(q^{2}\right)+N F_{n}\left(q^{2}\right)\right],
\end{aligned}
$$

where $\delta_{\mathrm{NSI}}=\epsilon_{\alpha \alpha}^{u V}=\epsilon_{\alpha \alpha}^{d V}(\alpha=e, \mu, \tau$ represents the neutrino flavor) denotes the NSI parameters. Equation (14) can be obtained from the more general NSIs (see, e.g., Refs. [40-42,44]) by neglecting the flavor-changing couplings $\epsilon_{\alpha \beta}^{q V}(\alpha \neq \beta)$ and assuming that the new flavorpreserving couplings $\left(\epsilon_{\alpha \alpha}^{q V}\right)$ are flavor symmetric for neutrinos and the first-generation quarks $(q=u, d)$. Then one can estimate the value of $\delta_{\text {NSI }}$ as

$$
\delta_{\mathrm{NSI}} \simeq-\frac{2 Z}{3 A} \Delta \sin ^{2} \theta_{W}^{*}=0.008,
$$

by assuming $F_{p}\left(q^{2}\right) \simeq F_{n}\left(q^{2}\right)$. These results indicate that the NSI contribution into the proton and neutron neutral current vector couplings is $3 \delta_{\mathrm{NSI}}=0.024$, which is even larger than the SM proton coupling $g_{V}^{p}=\frac{1}{2}-2 \sin ^{2} \theta_{W}^{\mathrm{SM}}=$ 0.02286 .

Moreover, we would like to point out that the deviation of $\sin ^{2} \theta_{W}^{*}$ from $\sin ^{2} \theta_{W}^{\mathrm{SM}}$ in neutrino physics can also potentially arise from the neutrino electromagnetic properties, e.g., the neutrino charge radius $\left\langle r_{\nu}^{2}\right\rangle$ [44-46]. Furthermore, the deviation could be as well from the dark parity violation $[35,47]$. All these scenarios beyond the SM can effectively shift the low-energy weak mixing angle in $\nu N$ interactions and worthy of further investigation with forthcoming more precise CE $\nu$ NS data in future. It will be also very interesting to check the similar effects in other weak neutral interaction measurements, e.g., APV and PREX.

Finally, it should be pointed out that the uncertainty of the extracted values for both $\Delta R_{n p}^{\mathrm{CsI}}$ and $\sin ^{2} \theta_{W}^{*}$ is very large due to the poor statistics of the current COHERENT data, and this hinders us from claiming a determination of the $\Delta R_{n p}^{\mathrm{CsI}}$ and $\sin ^{2} \theta_{W}^{*}$. Nevertheless, our results indicate that the $\Delta R_{n p}^{\mathrm{CsI}}$ is positively correlated with $\sin ^{2} \theta_{W}^{*}$ and the bestfit values lead to the possibility of significantly smaller values of $\Delta R_{n p}^{\mathrm{CsI}}$ and $\sin ^{2} \theta_{W}^{*}$ compared to the one-parameter fit to the COHERENT data with $\sin ^{2} \theta_{W}^{*}=\sin ^{2} \theta_{W}^{\mathrm{SM}}$. The present work thus suggests that the $\sin ^{2} \theta_{W}^{*}$ may play an important role in extracting neutron skin information from analyzing the CE $\nu$ NS data and a multidimensional fit is important in future analyses of high-precision CE $\nu$ NS data.

\section{SUMMARY AND OUTLOOK}

We have demonstrated that the low-energy effective weak mixing angle $\theta_{W}^{*}$ plays an important role in the extraction of neutron skin information of atomic nuclei from the $\mathrm{CE} \nu \mathrm{NS}$ experiments. By analyzing the $\mathrm{CE} \nu \mathrm{NS}$ data of the COHERENT experiment, we have found that while a one-parameter fit to the COHERENT data produces $\Delta R_{n p}^{\mathrm{CsI}} \simeq 0.68_{-1.13}^{+0.91} \mathrm{fm}$ with $\sin ^{2} \theta_{W}^{*}=\sin ^{2} \theta_{W}^{\mathrm{SM}}=0.23857$, a two-dimensional fit by varying $\Delta R_{n p}$ and $\sin ^{2} \theta_{W}^{*}$ leads to a strong positive correlation between $\Delta R_{n p}$ and $\sin ^{2} \theta_{W}^{*}$ with significantly smaller central values of $\Delta R_{n p}^{\mathrm{CsI}} \simeq 0.24_{-2.03}^{+2.30} \mathrm{fm}$ and $\sin ^{2} \theta_{W}^{*}=0.21_{-0.10}^{+0.13}$. While the best-fit value $\Delta R_{n p}^{\mathrm{CsI}} \simeq$ $0.24 \mathrm{fm}$ seems to be reasonable, the substantial deviation of the best-fit value $\sin ^{2} \theta_{W}^{*}=0.21$ from $\sin ^{2} \theta_{W}^{\mathrm{SM}}$ could give a hint on new physics in $\nu$-nucleon interactions.

Although the current large uncertainty does not allow us to claim a determination of the $\Delta R_{n p}^{\mathrm{CsI}}$ and $\sin ^{2} \theta_{W}^{*}$, our present work suggests that a multidimensional fit is important to extract useful information on neutron skin information (including its size and shape) and the low-energy effective 
$\sin ^{2} \theta_{W}^{*}$ from analyzing the high-precision data of future $\mathrm{CE} \nu \mathrm{NS}$ measurements. It will be also extremely interesting to explore the similar effects in other experiments of weak neutral interaction measurements.

\section{ACKNOWLEDGMENTS}

We thank Juan I. Collar for the useful communication on the quenching factor for CsI, Xiao-Gang $\mathrm{He}$ and Alexander I. Studenikin for useful discussions. This work was supported in part by the National Natural Science
Foundation of China under Grant No. 11625521, the Major State Basic Research Development Program (973 Program) in China under Contract No. 2015CB856904, the Program for Professor of Special Appointment (Eastern Scholar) at Shanghai Institutions of Higher Learning, Key Laboratory for Particle Physics, Astrophysics and Cosmology, Ministry of Education, China, and the Science and Technology Commission of Shanghai Municipality (11DZ2260700).
[1] B. A. Brown, Phys. Rev. Lett. 85, 5296 (2000); S. Typel and B. A. Brown, Phys. Rev. C 64, 027302 (2001).

[2] C. J. Horowitz and J. Piekarewicz, Phys. Rev. Lett. 86, 5647 (2001).

[3] R. J. Furnstahl, Nucl. Phys. A706, 85 (2002).

[4] S. Yoshida and H. Sagawa, Phys. Rev. C 69, 024318 (2004).

[5] L. W. Chen, B. A. Li, and C. M. Ko, Phys. Rev. C 72, 064309 (2005).

[6] B. G. Todd-Rutel and J. Piekarewicz, Phys. Rev. Lett. 95, 122501 (2005).

[7] M. Centelles, X. Roca-Maza, X. Viñas, and M. Warda, Phys. Rev. Lett. 102, 122502 (2009).

[8] L. W. Chen, C. M. Ko, B. A. Li, and J. Xu, Phys. Rev. C 82, 024321 (2010).

[9] X. Roca-Maza, M. Centelles, X. Viñas, and M. Warda, Phys. Rev. Lett. 106, 252501 (2011).

[10] B. K. Agrawal, J. N. De, and S. K. Samaddar, Phys. Rev. Lett. 109, 262501 (2012).

[11] J. M. Lattimer and M. Prakash, Science 304, 536 (2004); Phys. Rep. 442, 109 (2007).

[12] A. W. Steiner, M. Prakash, J. M. Lattimer, and P. J. Ellis, Phys. Rep. 411, 325 (2005).

[13] V. Baran, M. Colonna, V. Greco, and M. Di Toro, Phys. Rep. 410, 335 (2005).

[14] B. A. Li, L. W. Chen, and C. M. Ko, Phys. Rep. 464, 113 (2008).

[15] C. J. Horowitz, S. J. Pollock, P. A. Souder, and R. Michaels, Phys. Rev. C 63, 025501 (2001).

[16] T. Sil, M. Centelles, X. Viñas, and J. Piekarewicz, Phys. Rev. C 71, 045502 (2005).

[17] D. H. Wen, B. A. Li, and L. W. Chen, Phys. Rev. Lett. 103, 211102 (2009).

[18] H. Zheng, Z. Zhang, and L. W. Chen, J. Cosmol. Astropart. Phys. 08 (2014) 011.

[19] H. Zheng, K. J. Sun, and L. W. Chen, Astrophys. J. 800, 141 (2015).

[20] I. Angeli and K. P. Marinova, At. Data Nucl. Data Tables 99, 69 (2013).

[21] G. Fricke, C. Bernhardt, K. Heilig, L. A. Schaller, L. Schellenberg, E. B. Shera, and C. W. Dejager, At. Data Nucl. Data Tables 60, 177 (1995).
[22] F. Le Blanc et al., Phys. Rev. C 72, 034305 (2005).

[23] K. Kumar, R. Michaels, P. A. Souder, and G. M. Urciuoli, Spokespersons, http://hallaweb.jlab.org/parity/prex.

[24] S. Abrahamyan et al., Phys. Rev. Lett. 108, 112502 (2012).

[25] C. J. Horowitz et al., Phys. Rev. C 85, 032501(R) (2012).

[26] F. J. Fattoyev and J. Piekarewicz, Phys. Rev. Lett. 111, 162501 (2013).

[27] D. Akimov et al. (COHERENT Collaboration), Science 357, 1123 (2017).

[28] D. Z. Freedman, Phys. Rev. D 9, 1389 (1974).

[29] D. Z. Freedman, D. N. Schramm, and D. L. Tubbs, Annu. Rev. Nucl. Sci. 27, 167 (1977).

[30] M. Cadeddu, C. Giunti, Y. F. Li, and Y. Y. Zhang, Phys. Rev. Lett. 120, 072501 (2018).

[31] A. Trzcinska, J. Jastrzębski, P. Lubiński, F. J. Hartmann, R. Schmidt, T. von Egidy, and B. Kłos, Phys. Rev. Lett. 87, 082501 (2001).

[32] W. J. Swiatecki, A. Trzcinska, and J. Jastrzebski, Phys. Rev. C 71, 047301 (2005).

[33] C. Patrignani et al. (Particle Data Group), Chin. Phys. C 40, 100001 (2016).

[34] M. Tanabashi et al. (Particle Data Group), Phys. Rev. D 98 , 030001 (2018).

[35] K. S. Kumar, S. Mantry, W. J. Marciano, and P. A. Souder, Annu. Rev. Nucl. Part. Sci. 63, 237 (2013).

[36] S. G. Porsev, K. Beloy, and A. Derevianko, Phys. Rev. Lett. 102, 181601 (2009).

[37] V.A. Dzuba, J.C. Berengut, V. V. Flambaum, and B. Roberts, Phys. Rev. Lett. 109, 203003 (2012).

[38] B. M. Roberts, V. A. Dzuba, and V. V. Flambaum, Annu. Rev. Nucl. Part. Sci. 65, 63 (2015).

[39] D. Androić et al. (Jefferson Lab Qweak Collaboration), Nature (London) 557, 207 (2018).

[40] J. Billard, J. Johnston, and B. J. Kavanagh, J. Cosmol. Astropart. Phys. 11 (2018) 016.

[41] J. Barranco, O. G. Miranda, and T. I. Rashba, J. High Energy Phys. 12 (2005) 021.

[42] M. Lindner, W. Rodejohann, and X.-J. Xu, J. High Energy Phys. 03 (2017) 097.

[43] J. Liao and D. Marfatia, Phys. Lett. B 775, 54 (2017).

[44] D. K. Papoulias and T. S. Kosmas, Phys. Rev. D 97, 033003 (2018). 
[45] C. Giunti and A. Studenikin, Rev. Mod. Phys. 87, 531 (2015).

[46] M. Cadeddu, C. Giunti, K. A. Kouzakov, Y. F. Li, A. I. Studenikin, and Y. Y. Zhang, Phys. Rev. D 98, 113010 (2018).

[47] H. Davoudiasl, H.-S. Lee, and W. J. Marciano, Phys. Rev. Lett. 109, 031802 (2012); Phys. Rev. D 85, 115019 (2012).

[48] K. Patton, J. Engel, G. C. McLaughlin, and N. Schunck, Phys. Rev. C 86, 024612 (2012).

[49] B. C. Cañas, E. A. Garcés, O. G. Miranda, and A. Parada, Phys. Lett. B 784, 159 (2018).

[50] K. Scholberg (COHERENT Collaboration), Proc. Sci., NuFact2017 (2018) 020, [arXiv:1801.05546].

[51] M. Wang, G. Audi, F. G. Kondev, W. J. Huang, S. Naimi, and X. Xu, Chin. Phys. C 41, 030003 (2017).
[52] R. H. Helm, Phys. Rev. 104, 1466 (1956).

[53] H. De Vries, C. W. De Jager, and C. De Vries, At. Data Nucl. Data Tables 36, 495 (1987).

[54] R. W. Hasse and W. D. Myers, Geometrical Relationships of Macroscopic Nuclear Physics (Springer-Verlag, Heidelberg, 1988).

[55] J. Piekarewicz, A. R. Linero, P. Giuliani, and E. Chicken, Phys. Rev. C 94, 034316 (2016).

[56] J. Friedrich and N. Voegler, Nucl. Phys. A373, 192 (1982).

[57] D. Akimov et al. (COHERENT Collaboration), arXiv: 1804.09459.

[58] M. Warda, X. Viñas, X. Roca-Maza, and M. Centelles, Phys. Rev. C 81, 054309 (2010).

[59] C. M. Tarbert et al. (Crystal Ball at MAMI and A2 Collaborations), Phys. Rev. Lett. 112, 242502 (2014). 